\title{
A surprising treatment response in a patient with rare isolated growth hormone deficiency, type IB
}

\author{
Jordan Yardain Amar', Kimberly Borden², Elizabeth Watson ${ }^{3}$ and Talin Arslanian4 \\ ${ }^{1}$ Lewis Katz School of Medicine at Temple University, Philadelphia, Pennsylvania, USA, 2David Geffen School of \\ Medicine at UCLA, Los Angeles, California, USA, 3Sidney Kimmel College of Medicine at Thomas Jefferson University, \\ Philadelphia, Pennsylvania, USA, and 4Mattel Children's Hospital, University of California, Los Angeles, \\ California, USA
}

Correspondence should be addressed to J Y Amar

Email

tuf50857@temple.edu

\section{Summary}

Isolated Growth Hormone Deficiency (IGHD) is a rare cause of short stature, treated with the standard regimen of subcutaneous synthetic growth hormone (GH). Patients typically achieve a maximum height velocity in the first year of treatment, which then tapers shortly after treatment is stopped. We report a case of a 9-year-old male who presented with short stature ( $<3$ rd percentile for age and race). Basal hormone levels showed undetectable serum IGF1. Skeletal wrist age was consistent with chronologic age. Cranial MRI revealed no masses or lesions. Provocative arginine-GH stimulation testing demonstrated a peak GH level of $1.4 \mathrm{ng} / \mathrm{mL}$. Confirmatory genetic testing revealed a rare autosomal recessive single-nucleotide polymorphism (SNP) with mutational frequency of $2 \%$. GH supplementation was started and pursued for 2 years, producing dramatically increased height velocity. This velocity persisted linearly through adolescence, several years after treatment had been discontinued. Final adult height was $>95$ th percentile for age and race. In conclusion, this is a case of primary hypopituitarism with differential diagnosis of IGHD vs Idiopathic Short Stature vs Constitutional Growth Delay. This case supports two objectives: Firstly, it highlights the importance of confirmatory genetic testing in patients with suspected, though diagnostically uncertain, IGHD. Secondly, it demonstrates a novel secondary growth pattern with implications for better understanding the tremendous variability of GH treatment response.

\section{Learning points:}

- GHD is a common cause of growth retardation, and IGHD is a specific subtype of GHD in which patients present solely with short stature.

- The standard treatment for IGHD is subcutaneous synthetic GH until mid-parental height is reached, with peak height velocity attained in the 1st year of treatment in the vast majority of patients.

- Genetic testing should be strongly considered in cases of diagnostic uncertainty prior to initiating treatment.

- Future investigations of GH treatment response that stratify by gene and specific mutation will help guide treatment decisions.

- Response to treatment in patients with IGHD is variable, with some patients demonstrating little to no response, while others are 'super-responders.' 


\section{Background}

GHD is a well-documented cause of growth retardation in both males and females, and affects $1 / 3800$ children in the USA. It typically occurs with panhypopituitarism, presenting with low growth rate, hypoglycemia, microphallus, lethargy, and congenital blindness $(1,2)$. IGHD is a rare, specific subtype with deficiency only in $\mathrm{GH}$; therefore, patients present exclusively with short stature. The majority of cases are idiopathic, though 3-30\% of cases have shown traceable familial etiology. Mainstay treatment is subcutaneous synthetic GH injection until mid-parental height is reached. Because of the variability in clinical presentation and neurohormonal profile, clinical differentiation can be difficult. Further, the response to therapy ranges dramatically between patients. This case supports the use of confirmatory genetic testing in patients with suspected IGHD. Moreover, response to treatment is a key element to better understanding the pathogenesis of IGHD.

\section{Case presentation}

A 9-year-old male of Middle Eastern descent presented with a complaint of short stature relative to his peers. His mother reported that in a school with 87 males his age (as determined by school year), he was the shortest in stature. He otherwise had no history of developmental delay, reaching all developmental milestones at appropriate times (responding to name at 6 months, pulling-to-stand and waving 'bye' at 9 months, walking at 12 months). There was no history of birth injury (though he experienced a right inguinal herniation of his small intestine at 11 days of age that was surgically repaired; no complications of the surgery were reported) or significant head injury. Genealogically, his parents were not consanguineous. His mother's pregnancy was uneventful (birth weight: $3.62 \mathrm{~kg}$, s.D.: $0.3 \mathrm{~kg}, 71 \mathrm{st}$ percentile). Teacher reports described the patient's behavior as 'boisterous' but without behavioral misconduct.

On examination, the patient's height was $121.5 \mathrm{~cm}$ (3rd percentile for age and race). He showed no gross abnormalities on systemic examination. There were no dysmorphic features. He had no deficits in vision, motor coordination, or sensorineural hearing. Testicular volume (4.3 $\mathrm{mL}$ in each testes) and descent were normal. Head circumference was $51 \mathrm{~cm}$. The ratio of upper:lower segment span (1.12) suggested that his short stature was proportionate for males his age. Examination of secondary sexual characteristics revealed Tanner Stage II. Penis length was age-appropriate $(4.5 \mathrm{~cm})$.

\section{Investigation}

X-ray bone age studies of the left wrist were consistent with chronologic age and race. Initial screening with serum Insulin Growth Factor 1 (IGF1) was abnormally low (24.1 ng/mL). Basal Serum GH was also low $(0.02 \mathrm{ng} / \mathrm{mL})$. IGF Binding Protein 3 (IGF-BP3) was within normal limits $(4.6 \mathrm{mg} / \mathrm{L})$. Testosterone $(6.4 \mathrm{ng} / \mathrm{dL})$ and sex hormonebinding globulin (SHBG; $89 \mathrm{nmol} / \mathrm{L}$ ) were also within normal reference range. Adrenal function tests, thyroid function tests, liver function tests, and hemoglobin A1C were all normal (Table 1). 2-Plane cranial MRI showed no abnormalities. Mid-parental height was $150.25 \mathrm{~cm}$. Growth velocity from age 8 to 9 was determined as $1.3 \mathrm{~cm} /$ year. Provocative arginine-GH stimulation testing was undertaken during a 12-h fasting period. A standard in-house sex steroid priming protocol was followed prior to provocative testing as the patient's exam suggested pre-pubescent developmental stage. GH stimulation testing revealed a peak GH level of $1.4 \mathrm{ng} / \mathrm{mL}$ (lower limit of normal: $10 \mathrm{ng} / \mathrm{mL}$ ). Confirmatory molecular testing was sent for identification of possible mutations or copy-number repeats in genes GH1 and GHRH, and transcription factors LHX3, LHX4 and PROP1, POU1F1

Table 1 Baseline endocrine data.

\begin{tabular}{|c|c|c|c|}
\hline & Unit & Values & Reference Range \\
\hline $\mathrm{HbA} 1 \mathrm{C}$ & $\%$ & 4.6 & $4.3-5.8$ \\
\hline TSH & $\mu \mathrm{U} / \mathrm{mL}$ & 2.2 & $0.34-3.5$ \\
\hline fT4 & $\mathrm{ng} / \mathrm{dL}$ & 1.2 & $0.9-1.8$ \\
\hline fT3 & $\mathrm{pg} / \mathrm{mL}$ & 2.4 & $2.0-4.0$ \\
\hline ACTH & $\mathrm{pg} / \mathrm{mL}$ & 8.1 & $4.4-52$ \\
\hline Cortisol & $\mathrm{mg} / \mathrm{dL}$ & 4.3 & $2.7-15.5$ \\
\hline Basal GH & $\mathrm{ng} / \mathrm{mL}$ & 0.02 & $0-20$ \\
\hline Peak GH & $\mathrm{ng} / \mathrm{mL}$ & 1.4 & $>5$ \\
\hline IGF1 & $\mathrm{ng} / \mathrm{mL}$ & 24 & $68-216$ \\
\hline IGF-BP3 & $\mathrm{mg} / \mathrm{L}$ & 4.6 & $1.8-7.1$ \\
\hline LH & IU/mL & 5.1 & $1.6-9.5$ \\
\hline FSH & IU/mL & 7.2 & $1.2-15$ \\
\hline PRL & $\mathrm{ng} / \mathrm{mL}$ & 1.3 & $1.2-15$ \\
\hline Test & $\mathrm{ng} / \mathrm{dL}$ & 6.4 & $<7-20$ \\
\hline SHBG & $\mathrm{nmol} / \mathrm{L}$ & 89 & $49-179$ \\
\hline NSB & $\%$ & 0.4 & $<8$ \\
\hline FIRI & $\mu \mathrm{U} / \mathrm{mL}$ & 4.8 & $1.7-10.4$ \\
\hline TIRI & $\mu \mathrm{U} / \mathrm{mL}$ & 5.6 & $5.0-15$ \\
\hline
\end{tabular}

ACTH, adrenocorticotrophic hormone; FIRI, free immunoreactive insulin; FSH, follicle stimulating hormone; fT3, free triiodothyronine; fT4, free thyroxine; GH, growth hormone; HbA1c, hemoglobin A1C; IGF1, insulin-like growth factor-I; LH, luteinizing hormone; NSB, non-specific binding; $\mathrm{PRL}$, prolactin; peak $\mathrm{GH}$, peak stimulated growth hormone; SHBG, sex hormone-binding globulin; test, testosterone; TIRI, total immunoreactive insulin; TSH, thyroid stimulating hormone. 
and HESX1. Mutational analysis revealed a 5'IVS-4 single nucleotide (Guanine-to-Cytosine) nonsense mutation in the $17 \mathrm{q} 23.3$ locus. A literature review indicated that this mutation has been attributed exclusively to IGHD, type IB (3). Accordingly, the patient was diagnosed with this specific subtype. Additional genetic testing was performed in the parents and living grandparents, which identified each parent and 2 grandparents as heterozygotic carriers. The paternal grandfather was unavailable for testing. The maternal grandmother was deceased.

\section{Treatment}

After obtaining parental and patient consent, oncedaily subcutaneous administration of synthetic GH was started at age 9 years and 322 days, and was discontinued 412 days later. During that time, dosage was titrated to weight $(0.18 \mathrm{mg} / \mathrm{kg} /$ week $)$ at every three-month visit. Serum IGF1 was measured at these intervals to verify that dosing remained within a clinically therapeutic range. At no point during the treatment window did IGF1 exceed the age-adjusted upper limit of normal. Repeat IGF1 screening at ages $14(102 \mathrm{ng} / \mathrm{mL})$ and $15(132 \mathrm{ng} / \mathrm{mL})$ were also within appropriate range.

\section{Outcome and follow-up}

Body height increased to $152.7 \mathrm{~cm}$ (62nd percentile) by the end of the treatment period (Fig. 1). Body weight increased from $26.7 \mathrm{~kg}$ (33rd percentile) to $41.6 \mathrm{~kg}$ (50th percentile). Height velocity during the 1st year of treatment was $8.1 \mathrm{~cm} /$ year. Treatment was stopped

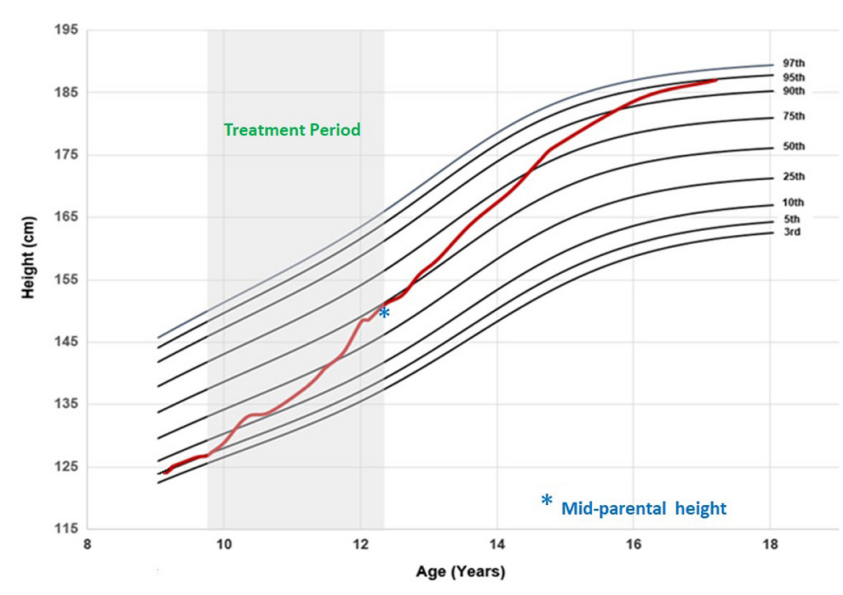

Figure 1

Patient's height plotted against CDC-normed growth chart for boys. Grey window denotes the GH treatment period. Red axis denotes the patient's height.

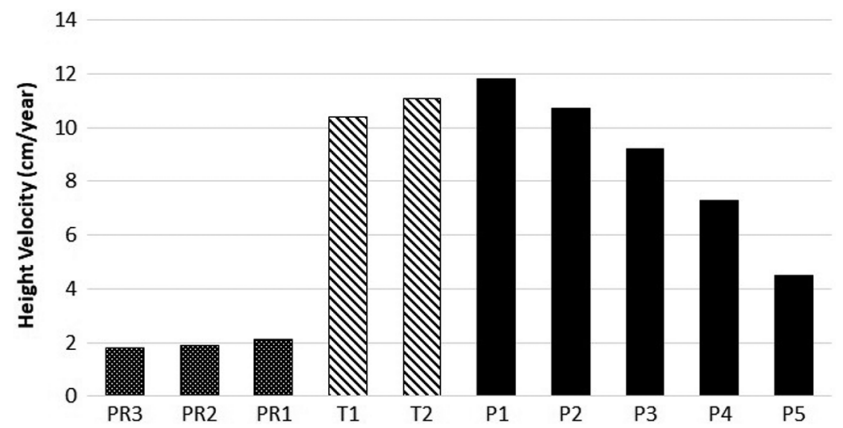

Figure 2

Height velocity with respect to treatment year. P1, post-treatment year 1; $\mathrm{P} 2$, post-treatment year 2; P3, post-treatment year 3; P4, post-treatment year 4; P5, post-treatment year 5; PR1, pre-treatment year 1; PR2, pre-treatment year 2; PR3, pre-treatment year 3; T1, treatment year 1; T2, treatment year 2 .

shortly after age 12, when the mid-parental height was reached. The patient's height on the last day of treatment was $150.3 \mathrm{~cm}$ (51st percentile). Following cessation of treatment, rapid linear growth continued into adulthood (Fig. 1). Final adult height was $186.8 \mathrm{~cm}$ (96th percentile). Final weight at this point in time was $72.4 \mathrm{~kg}$ (66th percentile). Sexual maturation continued at age-appropriate milestones (Tanner Stage 3: 12.6 years, Tanner Stage 4: 14.5 years, Tanner Stage 5: 15.7 years). Height velocities during the first and second year following treatment were 10.4 and $11.1 \mathrm{~cm} /$ year, respectively. Height velocities for the 5 consecutive years following cessation of treatment were 11.8, 10.7, and 9.2, 7.3 , and $4.5 \mathrm{~cm} /$ year, respectively (Fig. 2).

\section{Discussion}

This case highlights a rare cause of primary hypopituitarism with a broad differential diagnosis. Idiopathic Short Stature was considered as a possible cause, but was unlikely as the patient demonstrated undetectable levels of serum IGF-1 on repeat testing prior to beginning therapy. Direct pituitary damage (e.g. traumatic brain injury, Pituitary Apoplexy) was considered as possible etiologies in retrospective analyses. However, there was no history of trauma, birth complications, or ingestion of toxic substances, and MRI revealed no corresponding lesion. We considered the possibility of unrecognized maternal alcohol consumption, but it is unlikely since the patient did not have any of the pathognomonic facial features of Fetal Alcohol Syndrome (low nasal bridge, epicanthal folds, and micrognathia). Moreover, alcohol consumption has only been identified in the literature as a cause of panhypopituitarism rather than IGHD (4). Suspicion of 
neurological damage secondary to maternal ingestion of other toxins was low as the patient demonstrated no other physical abnormalities or cognitive deficits on evaluation, both of which are typical sequaele (5). Nutritional deficit was not suspected given serum albumin and prealbumin levels were within normal reference ranges and proportionate weight change prior to treatment.

Lastly, given the singular complaint of short stature, we considered Constitutional Growth Delay. It is wellknown that up to $70 \%$ of children with isolated GHD will not have persistent GHD into adolescence or adulthood (6). Accordingly, we remained wary of initiating GH treatment without clear indication. Patients with Constitutional Growth Delay tend to resume normal height velocities by ages 2-3 and a delayed skeletal age (as estimated by wrist $\mathrm{X}$-ray), commonly $2-4$ years behind chronological age $(7,8)$. Our patient demonstrated low height velocity through age 9 , and his skeletal age was consistent with chronological age. However, these trends are by no means definitive. Moreover, both IGHD and Constitutional Growth Delay may show poor response to provocative testing (9), calling into question the inter-trial reliability of testing (10). Given the clinical equipoise, we elected for genetic testing. The 5'IVS-4 single nucleotide (Guanine-to-Cytosine) nonsense SNP has a mutational frequency of $\sim 2 \%$ and presents with mild-to-severe short stature without dysmorphies or neuroendocrine derangements (3).

IGHD, type IB is characterized by autosomal recessive inheritance, a severely diminished endogenous GH pattern, and a very low but detectable peak GH stimulatory test (11). This endocrine profile matched that of our patient. Moreover, genetic testing of the parents identified a phylogenetically traceable, heterogenous mutation in each parent, indicative of autosomal inheritance. In this particular case, genetic testing allowed for definitive diagnosis of IGHD, type IB. While some clinicians may argue in favor of close monitoring of height velocity for a self-correcting growth delay, mathematical modeling has identified age of treatment onset as one of the strongest predictors of treatment response (12). Delaying treatment would have possibly diminished the patient's therapeutic outcome. Recent work by Khadilikar et al. showed that in children matched for age and baseline height, treatment response to GH in those with homozygous GH1 deletions was poor relative to those with a GHRHR mutation or without any known mutation (13). In cases of previously-identified 'poor responders,' genetic testing may save families from ultimately fruitless and expensive therapies. Often, screening of the parents is sufficient, though in our patient's case, we elected to pursue additional family members due to the rarity of this particular mutation.

Our own patient represents the other end of the spectrum of the treatment response: a 'super-responder.' Though type IB is typically a severe form of GHD, treatment response with lower levels of GH show reasonable response, while avoiding development of anti-GH antibodies (14).

However, treatment response can vary significantly and is not well-characterized $(2,14)$. These potential limitations were discussed with the family, who elected to proceed regardless. While reasonable to expect some efficacy, the phenomenon observed in our patient was fairly surprising. We considered that this sustained growth rate may simply be a pubertal surge. However, even in healthy males, growth continues asymptotically as of age 15 at a rate roughly half of that observed in our patient (15). Our patient's observed height velocities far exceeded the standardized height velocities, which were first characterized by Backer et al. (16), and validated by others $(17,18)$. Moreover, to our knowledge, there is no other reported case of IGHD of any subtype where height velocity after treatment exceeded that of the 1st year of treatment.

Based on these surprising observations, we conclude that the range of treatment response in patients with IGHD may vary far more than previously understood. With an increasing number of source mutations being identified, future investigations of treatment efficacy would likely benefit from greater stratification by genetic mutation.

Declaration of interest

All authors declare that there is no conflict of interest that could be perceived as prejudicing the impartiality of the research reported.

\section{Funding}

This research did not receive any specific grant from any funding agency in the public, commercial or not-for-profit sector.

\section{Patient consent}

Informed patient consent was obtained for publication in this journal. Our patient was a legal adult at the time of manuscript preparation, and was able to sign consent for himself. 


\section{Patient's perspective}

Our patient declined to submit a patient perspective, but he did communicate to us that reviewing our work and his case inspired his own desire to pursue a career in translational research.

\section{Author contribution statement}

J A was responsible for drafting the manuscript and data synthesis. K B was responsible for drafting the manuscript and literature review. E W was responsible for creating the code used to generate figures and literature review. T A was the supervising physician responsible for clinical accuracy and reviewing the manuscript.

\section{References}

1 Persani L \& Bonomi M 2017 The multiple genetic causes of central hypothyroidism. Best Practice and Research Clinical Endocrinology and Metabolism 31 255-263. (doi:10.1016/j.beem.2017.04.003)

2 Di Iorgi N, Morana G, Allegri A, Napoli F, Gastaldi R, Calcagno A, Patti G, Loche S \& Maghnie M 2016 Classical and non-classica causes of GH deficiency in the paediatric age. Best Practice and Research Clinical Endocrinology and Metabolism 30 705-736. (doi:10.1016/j.beem.2016.11.008)

3 Giordano M 2016 Genetic causes of isolated and combined pituitary hormone deficiency. Best Practice and Research Clinical Endocrinology and Metabolism 30 679-691. (doi:10.1016/j.beem.2016.09.005)

4 Blaine S \& Sinha R 2017 Alcohol, stress, and glucocorticoids: from risk to dependence and relapse in alcohol use disorders. Neuropharmacology 122 136-147. (doi:10.1016/j. neuropharm.2017.01.037)

5 Watson A, Ellington S, Nelson C, Treadwell T, Jamieson D \& Meaney-Delman D 2017 Preparing for biological threats: addressing the needs of pregnant women. Birth Defects Research 109 391-398. (doi:10.1002/bdr2.1016)

6 Zhu J \& Chan Y 2017 Adult consequences of self-limited delayed puberty. Pediatrics 139 e20163177. (doi:10.1542/peds.2016-3177)

7 Soliman A \& Sanctis V 2012 An approach to constitutional delay of growth and puberty. Indian Journal of Endocrinology and Metabolism 16 698. (doi:10.4103/2230-8210.100650)

8 Rothermel J, Lass N, Toschke C \& Reinehr T 2016 Progressive decline in height standard deviation scores in the first 5 years of life distinguished idiopathic growth hormone deficiency from familial short stature and constitutional delay of growth. Hormone Research in Paediatrics 86 117-125. (doi:10.1159/000448283)
9 Gunn KC, Cutfield WS, Hofman PL, Jefferies CA, Albert BB \& Gunn AJ 2014 Constitutional delay influences the auxological response to growth hormone treatment in children with short stature and growth hormone sufficiency. Scientific Reports 46061. (doi:10.1038/srep06061)

10 Harrington J \& Palmert MR 2012 Clinical review: Distinguishing constitutional delay of growth and puberty from isolated hypogonadotropic hypogonadism: critical appraisal of available diagnostic tests. Journal of Clinical Endocrinology and Metabolism 97 3056-3067. (doi:10.1210/jc.2012-1598)

11 Pérez Jurado LA \& Argente J 1994 Molecular basis of familial growth hormone deficiency. Hormone Research 42 189-197. (doi:10.1159/000184192)

12 Quigley C, Zagar A, Liu C, Brown D, Huseman C, Levitsky L, Repaske DR, Tsalikian E \& Chipman JJ 2013 United States multicenter study of factors predicting the persistence of GH deficiency during the transition period between childhood and adulthood. International Journal of Pediatric Endocrinology 20136 (doi:10.1186/1687-9856-2013-6)

13 Khadilkar V, Phadke N, Khatod K, Ekbote V, Gupte S, Nadar R \& Khadilkar A 2017 Molecular genetics of growth hormone deficient children: correlation with auxology and response to first year of growth hormone therapy. Journal of Pediatric Endocrinology and Metabolism 30 669-675. (doi:10.1515/jpem-2016-0382)

14 Aguiar-Oliveira MH, Anita HO, Souza AHO, Oliveira CRP, Campos VC, Oliveira-Neto LA \& Salvatori R 2017 Mechanisms in endocrinology. The multiple facets of GHRH/GH/IGF-I axis: lessons from lifetime, untreated, isolated GH deficiency due to a GHRH receptor gene mutation. European Journal of Endocrinology 177 R85-R97. (doi:10.1530/EJE-16-1047)

15 2012-2014 Growth Hormone Deficiency. Chiswick London: UK Child Growth Foundation.

16 Bakker B, Frane J, Anhalt H, Lippe B \& Rosenfeld R 2008 Height velocity targets from the national cooperative growth study for firstyear growth hormone responses in short children. Journal of Clinical Endocrinology and Metabolism 93 352-357. (doi:10.1210/jc.2007-1581)

17 Ranke M, Lindberg A, Brosz M, Kaspers S, Loftus J, Wollmann H, Kołtowska-Haggstrom M \& Roelants M 2012 Accurate long-term prediction of height during the first four years of growth hormone treatment in prepubertal children with growth hormone deficiency or turner syndrome. Hormone Research in Paediatrics 78 8-17. (doi:10.1159/000339468)

18 Kaplowitz P, Shulman D, Frane J, Jacobs J \& Lippe B 2013 Characteristics of children with the best and poorest first- and second-year growth during rhGH therapy: data from 25 years of the Genentech national cooperative growth study (NCGS). International Journal of Pediatric Endocrinology 2013 9. (doi:10.1186/1687-9856-2013-9)

Received in final form 8 September 2017

Accepted 19 September 2017 